\title{
Habitat-related birdsong divergence: a multi-level study on the influence of territory density and ambient noise in European blackbirds
}

\author{
Erwin A. P. Ripmeester • Jet S. Kok • \\ Jacco C. van Rijssel • Hans Slabbekoorn
}

Received: 6 August 2009 /Revised: 2 September 2009 /Accepted: 3 September 2009/Published online: 30 September 2009

(C) The Author(s) 2009. This article is published with open access at Springerlink.com

\begin{abstract}
Song plays an important role in avian communication and acoustic variation is important at both the individual and population level. Habitat-related variation between populations in particular can reflect adaptations to the environment accumulated over generations, but this may not always be the case. In this study, we test whether variation between individuals matches local conditions with respect to noise level and territory density to examine whether short-term flexibility could contribute to song divergence at the population level. We conducted a case study on an urban and forest population of the European blackbird and show divergence at the population level (i.e. across habitats) in blackbird song, anthropogenic noise level and territory density. Unlike in several other species, we found a lack of any correlation at the individual level (i.e. across individuals) between song features and ambient noise. This suggests species-specific causal explanations for noisedependent song differentiation which are likely associated with variation in song-copying behaviour or feedback constraints related to variable singing styles. On the other hand, we found that at the level of individual territories, temporal features, but not spectral ones, are correlated to territory density and seasonality. This suggests that shortterm individual variation can indeed contribute to habitatdependent divergence at the population level. As this may undermine the potential role for song as a population marker, we conclude that more investigations on individual song flexibility are required for a better understanding of the
\end{abstract}

Communicated by W. Searcy

E. A. P. Ripmeester $(\bowtie) \cdot J$. S. Kok · J. C. van Rijssel •

H. Slabbekoorn

Sylvius Laboratory, Behavioural Biology, IBL, Leiden University, P.O. Box 9505, 2300 RA Leiden, The Netherlands

e-mail: e.a.p.ripmeester@biology.leidenuniv.nl impact of population-level song divergence on hybridisation and speciation.

Keywords Habitat-related song divergence .

Acoustic variation $\cdot$ Anthropogenic noise $\cdot$ Urbanisation .

Territory density $\cdot$ Season

\section{Introduction}

Geographic song variation among different populations of the same species is a widespread phenomenon among songbird species (Mundinger 1982; Kroodsma 2004). Especially, habitat-related variation may be critical to mate recognition and has been attributed a potentially important role in the process of speciation (Slabbekoorn and Smith 2002a; Podos and Nowicki 2004). Habitat-related variation may concern evolutionary adaptations accumulated over generations. However, it has been suggested that it can also emerge as a result of more short-term changes within an individual's lifetime in response to exposure to local circumstances that differ between habitats (Slabbekoorn and Peet 2003; Slabbekoorn and den Boer-Visser 2006). It is important to understand whether adaptations, accumulated over generations, or short-term flexibilities are underlying habitat-related acoustic variation as song divergence totally attributable to ontogenetic or immediate flexibility is less likely to affect hybridisation and speciation events (Slabbekoorn and Smith 2002a; Ellers and Slabbekoorn 2003).

Several environmental selection pressures can shape birdsong characteristics. Transmission properties as well as the spectral overlap and loudness of the background noise determine which frequencies are suitable for communication (Morton 1975; Brumm and Slabbekoorn 2005). 
Anthropogenic noise, which consists of relatively lowfrequency sounds, is for example known to affect song variation at the individual level. Urban great tits (Parus major), song sparrows (Melospiza melodia) and house finches (Carpodacus mexicanus) sing with a higher minimum frequency in territories with high levels of anthropogenic noise (Slabbekoorn and Peet 2003; Fernández-Juricic et al. 2005; Wood and Yezerinac 2006). Furthermore, a study on great tits at the population level revealed a consist pattern of divergence in frequency use between urban and forest great tits which is most likely due to the impact of anthropogenic noise (Slabbekoorn and den Boer-Visser 2006). Similar noise-dependent song variation has also been found in nonurban contexts within species living in forest types with strikingly different noise profiles (Slabbekoorn and Smith 2002b; Dingle et al. 2008). The noise-dependent variation between individuals may reflect short-term flexibility within individuals and suggests that song variation matching ambient noise profiles at the population level may not always be exclusively attributed to long-term adaptations.

Habitats may not only differ acoustically, but may also differ in bird density, related for example to the abundance of food or nest sites. An indirect effect of habitat on bird song through variation in territory density is likely related to the associated differences in the number of social interactions. It has been shown for several bird species that territorial interactions can induce changes in individual song characteristics (Nelson 1984; Capp and Searcy 1991; Martin-Vivaldi et al. 2004). Consequently, variation in intensity of territorial interactions between individuals related to differences between habitats in territory density may potentially explain population differences in song. A study on willow warblers (Phylloscopus trochilus) showed that males in a high-density population produced on average more "double songs" and more notes in the initial phrase of songs (Goretskaia 2004). Goretskaia also speculates that these differences can be attributed to densityrelated mean level of arousal.

Both noise- and density-dependent song-type selection and singing style may have an impact on population-level divergence patterns via short-term flexibility. Insight into the causes of variation at the individual level is, therefore, required to properly interpret variation at the population level. Acoustic variation at both levels may concern spectral as well as temporal parameters (Slabbekoorn and den Boer-Visser 2006; c.f. Ripmeester et al. unpublished). As both noise levels and territory density may theoretically vary in concert between and within habitats, studies on noise-related variation ought to take the factor density into account. We, therefore, conducted a multi-level study in which the influence of ambient noise as well as territory density was investigated at the individual and population level (see e.g. Fernández-Juricic et al. 2009).
European blackbirds (Turdus merula) occur at higher densities in cities than in forests (Luniak et al. 1990; Clement and Hathway 2000) and are known to vary acoustically both at the population level between urban and forest environment (c.f. Ripmeester et al. unpublished) and at the individual level related to agonistic context (Dabelsteen and Pedersen 1990; Ripmeester et al. 2007). Male blackbirds have a large repertoire of songs with a stereotypic structure whereby each song starts with a motif part consisting of relatively simple low-frequency elements followed by a twitter part having more variable high-frequency elements (Fig. 1, Dabelsteen and Pedersen 1985; Ripmeester et al. 2007). Urban males produce songs with higher peak frequencies for both the motif and twitter part and have larger twitter proportions (i.e. a longer twitter relative to the motif within a song) and longer intra-song pauses (c.f. Ripmeester et al. unpublished). The level of arousal associated with territorial interactions has been suggested to raise the proportion of twitter within a song and to reduce the pause duration between songs (Dabelsteen 1984; Dabelsteen and Pedersen 1990). A recent test of this hypothesis led to equivocal results, but added another parameter varying with context: an increased frequency of the twitter part associated with an agitated response (Ripmeester et al. 2007).

The aim of this study is to investigate habitat-related birdsong divergence and examine the potential impact of short-term individual variation caused by local and current circumstances that may vary consistently between habitats. We use an integrated analysis to examine the effect of ambient noise and territory density on both variation at the population level (i.e. across habitats) and variation at the individual level (i.e. among individuals). For this purpose, we compare songs, noise levels and territory density of European blackbirds from a city and a nearby forest in the Netherlands.

\section{Materials and methods}

Study species and area

The European blackbird (T. merula) used to live in forests and rural areas, but it has expanded its range into cities in large parts of Europe since the early nineteenth century (Luniak et al. 1990; Clement and Hathway 2000). They are currently thriving in Dutch cities and forests making them the most numerous breeding species in the Netherlands with over one million breeding pairs (Hustings and Vergeer 2002). Blackbirds are songbirds, and males sing in the entire breeding season, which lasts roughly from February to the beginning of August, during which they regularly sing throughout the day with a peak in activity level around dawn and dusk (de Vos and de Meersman 2005). 


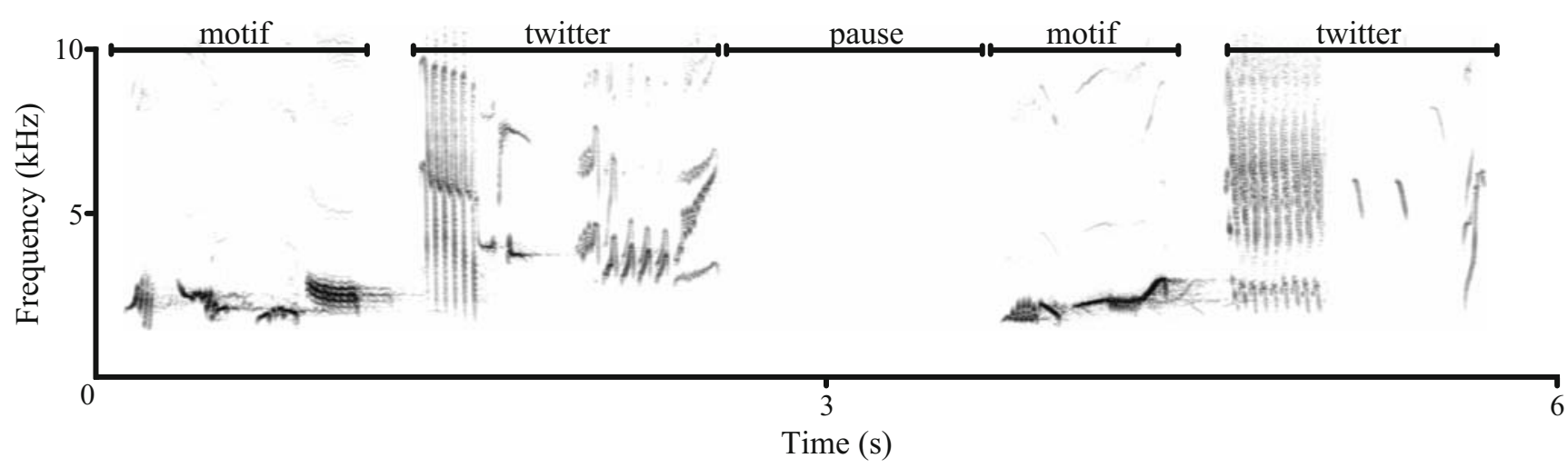

Fig. 1 Spectrogram showing the stereotypic singing style of blackbirds. Each song starts with a motif part followed by a twitter part and there is a short pause without sound in between two consecutive songs

We conducted our study in the city of Leiden $\left(52^{\circ} 09^{\prime} \mathrm{N}\right.$, $\left.4^{\circ} 29^{\prime} \mathrm{E}\right)$ and the forest-dune area of Meijendel $\left(52^{\circ} 08^{\prime} \mathrm{N}\right.$, $\left.4^{\circ} 20^{\prime} \mathrm{E}\right)$, which were both part of a previous study (c.f. Ripmeester et al. unpublished). Leiden is a relatively old medium-sized Dutch city with 117,000 inhabitants. Meijendel is located in the south of a large old mixed-forest dune area and has a size of approximately 1,875 ha without any large roads in its vicinity. The centres of Leiden and Meijendel are approximately $10 \mathrm{~km}$ apart, and the two areas are separated by about $4 \mathrm{~km}$ of agricultural area and the village of Wassenaar (Fig. 2). Recordings of urban birds were made in and around the city centre of Leiden. Forest blackbirds were recorded in patches of forests of Meijendel, which were well spread over the entire area. We avoided areas where blackbirds had been recorded in the previous study.

\section{Song recording and analysis}

Song recordings were made during the dawn chorus on rainless weekdays with little or no wind between the 29th of March and the 7th of June 2007. The dawn chorus occurs every morning starting with a sudden burst of singing activity during which all territorial male blackbirds sing simultaneously for approximately 30 to $45 \mathrm{~min}$. We recorded singing males that were not direct neighbours of each other and approached them up to a distance of 10 to $15 \mathrm{~m}$. Recordings were made with a Marantz PMD670 digital recorder in wave format files with a sampling rate of $48.0 \mathrm{kHz}$ using a Sennheiser ME67/K6 directional microphone placed in a wind basket with hairy cover attached to a pistol grip (Sennheiser MZ 70-1 set).

We analysed ten songs and ten intra-song pauses for each individual with Signal version 3.1.1. Spectrograms were made with a Fast Fourier transformation size of 512, a high-pass filter of $0.6 \mathrm{kHz}$ and the rainbow colour palette setting with a low and high intensity threshold of -35 and $-15 \mathrm{~dB}$, respectively. The duration of the motif and the twitter were measured manually by cursor placements on a computer screen showing spectrograms with a frequency range between 0 and $14 \mathrm{kHz}$ on the $y$-axis and $5 \mathrm{~s}$ on the $x$-axis, resulting in a temporal resolution of $7 \mathrm{~ms}$. The twitter proportion of a song was calculated from the duration of the motif and twitter. Pause duration between two consecutive songs was measured in a similar way except that spectrograms on the screen instead showed $10 \mathrm{~s}$ of recording on the $x$-axis giving a temporal resolution of $14 \mathrm{~ms}$. Furthermore, the peak frequencies of the motif and the twitter were determined by averaging the loudest frequency present in five time segments of equal duration of the motif or twitter (Slabbekoorn and Smith 2002b). The spectral resolution of the peak frequency per segment was $94 \mathrm{~Hz}$.

\section{Ambient noise}

We measured ambient noise levels in each territory immediately after the song recording. These noise measurements were used to make a general comparison between noise levels of urban and forest territories during the dawn chorus in the study period. There is a seasonal change in traffic noise levels related to a shift in time of sunrise with the season. There is little traffic on the road early in the morning, but it rapidly increases when people start to go to work. Birds started to sing at 5:15 A.M. in the city and 6:30 A.M. in the forest at the beginning of our field season, but the starting time at the end of the season was 4:00 A.M. and 4:45 A.M. in the city and forest, respectively. A pilot study in 2006 showed that the level of low-frequency noise during the dawn chorus decreases during the season in the city, but not in the forest as there is hardly any traffic there anyway. We made additional noise measurements in territories in the city during the rush hour from 7:30 A.M. until 8:30 A.M. between the 4th and 8th of June 2007. The noise measurements during the rush hour and the dawn chorus are used as estimates for noise conditions relative to other territories in the city and forest, respectively. 
Fig. 2 Illustration of the study areas. The schematic map at the top of the illustration shows an overview of the locations of the two study areas, Meijendel and Leiden. Barred areas indicate urban habitat, and the light-grey area is forest-dune habitat. The left and right maps at the bottom of the illustration are satellite images of parts of the study areas in Meijendel and Leiden, respectively. Circles show territories in which blackbirds were recorded. The colours of the circles represent the anthropogenic noise level in the territories measured during the dawn chorus in Meijendel and between 7:30 A.M. and 8:30 A.M in Leiden (see the "Materials and methods" section for details). The number of lines attached to the circles represents the number of neighbours around the territory that were heard singing during the dawn chorus (see the "Materials and methods" section for details)
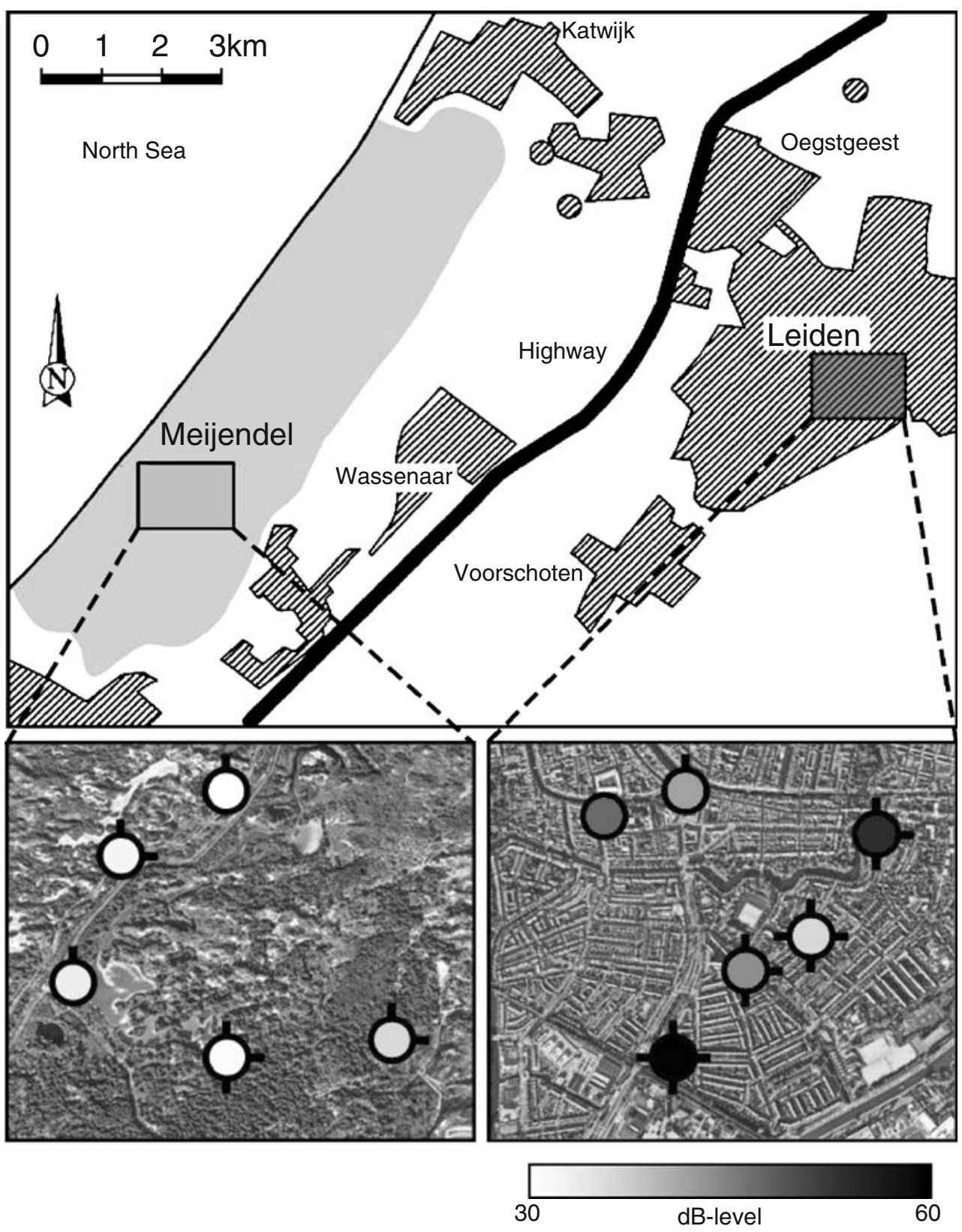

Noise measurements of $2 \mathrm{~min}$ were made in every territory at the position where the focal male was recorded using the same microphone and recording equipment as for the song recordings. The microphone was held horizontally at a height of about $1.5 \mathrm{~m}$. After every $30 \mathrm{~s}$, the recording was paused for several seconds, during which the microphone was turned $90^{\circ}$ clockwise to get recordings from four directions. We removed those moments in the noise recording in which the focal male was singing using Avisoft-SAS Lab-Pro version 4.3 (Avisoft Bioacoustics, Berlin, Germany) by looking at spectrograms with a FFT size of 512 with $50 \%$ overlap and a Hamming window. These moments were easily identified as a second microphone (Sennheiser ME67) was continuously pointed towards the focal male and recorded song simultaneously on the second channel of the stereo recorder.

We calculated the root mean square (RMS) values of the $1.0 \mathrm{kHz}$ octave band in the noise wave files using Matlab version 7.5 (the Mathworks, Inc.). The amount of energy in the $1.0 \mathrm{kHz}$ octave band reflects the level of low-frequency anthropogenic noise in a territory. The RMS values could be transformed into decibel levels as the microphone and recorder were calibrated. The calibration was done by playing white noise via a Vifa MG10SD09-08 speaker in an isolation chamber at various decibel levels. The playback was set to a volume level of 40 to $90 \mathrm{~dB}(Z)$ (reference level $20 \mu \mathrm{Pa}$ ) with intervals of $5 \mathrm{~dB}(\mathrm{Z})$ using a Pulsar Model 30 SPL metre $0.5 \mathrm{~m}$ away from the speaker. The RMS 
values of the white noise playbacks with known decibel levels made it possible to transform all RMS values of the field recordings into decibel values. The decibel levels of microphone recordings are roughly $15 \mathrm{~dB}$ below the $\mathrm{db}(\mathrm{Z})$ scale of the SPL metre due to a higher directionality of the microphone relative to the SPL metre (see Slabbekoorn et al. 2007 for the impact of recording directionality on amplitude measurements). Nevertheless, the decibel scale we use in this study makes it possible to compare noise levels between territories.

\section{Territory density}

Singing blackbirds within a distance of $100 \mathrm{~m}$ are expected to hear each other properly, and vocal communication at this distance is likely to play a role in territorial defence. We, therefore, choose the number of blackbirds singing within a range of $100 \mathrm{~m}$ of a recorded territorial male during the dawn chorus as a proxy for the level of relevant social singing interactions of a focal male.

There are often more objects blocking sound between a singing blackbird and an observer on the ground than between two blackbirds that are both positioned relatively high. A human observer is, therefore, likely to miss some singing birds which the focal bird can hear. This will happen more often in the city than in the forest as there are many buildings in urban areas whereas forests are relatively open. We calculated a corrected territory density measure to take this problem into account using the programme Distance version 5.0. This programme calculates the probabilities of detecting a singing bird at varying distances based on the difference between the observed and expected distribution of distances between focal birds and detected birds in its vicinity. The expected distribution assumes a linear relationship between the number of detected singing blackbirds and the size of the area around the position of the focal bird. The observed distribution was acquired in two steps. Firstly, one observer counted for $1 \mathrm{~min}$ the singing blackbirds that were audible from the position where the focal male was simultaneously being recorded by another observer. Secondly, all detected singing males were located, and their position was determined relative to the focal male with a GPS (Garmin eTrex Venture) and the programme EasyGPS version 2.3.8. This procedure took just several minutes, and more than $95 \%$ of the singing birds were located before they stopped singing. Data of focal birds for which the density measurement could not be completed in time were not included in further analyses.

Modelling detection probability curves on the observed distribution was done with the setting "Hazard key function with cosine series expansion" using sequential model selection based on the lowest Akaike Information Criterion.
The modelling showed that the human observer missed $11 \%$ and $22 \%$ of singing blackbirds within a radius of $100 \mathrm{~m}$ in the forest and city, respectively. We, therefore, corrected for the observer bias by adding $11 \%$ to the number of detected singing blackbirds within $100 \mathrm{~m}$ of the focal male in the forest and $22 \%$ in the city. The outcomes of statistical analyses were not affected by these corrections. For reasons of clarity and precision we only report results of the corrected density measure, which we refer to as "territory density" from here onwards.

\section{Statistical analysis}

Statistical analyses were conducted in $R$ version 2.6.2. (Ihaka and Gentleman 1996). Territory density and noise levels between habitats were compared with Wilcoxon rank-sum tests. We used linear mixed models (LMM) for tests involving song. Measurements of all songs were included in models, and intra-individual variation was taken into account by including the variable "individual" as a random factor. The variable "habitat" (city vs. forest) was entered as a fixed factor into models when data from both the city and the forest were included in one analysis. This was not the case for models testing the effect of noise on song, because these analyses were done for the city and forest separately. The noise levels of the $1 \mathrm{kHz}$ octave band, measured either during the dawn chorus in the forest or later in the morning in the city, were used as a fixed factor "noise". We tested the relationship between territory density and song by including the fixed factor "territory density" in models. Models testing for the effect of territory density were performed on the combined data of the city and the forest as well as per habitat when noise levels were included in the analysis. Data from a related study suggests that particularly temporal song characteristics change during the breeding season (c.f. Ripmeester et al. unpublished). We, therefore, included the fixed factor "Julian date" in all models. Model selection was based on stepwise removal of non-significant factors using 0.05 as the critical value of the deletion $p$ value. We visually verified model assumptions of normally distributed errors and absence of heteroscedasticity in the minimal adequate model.

\section{Results}

\section{Habitat differences}

We measured song, noise and density characteristics in 27 and 24 territories of, respectively, urban and forest males. We found habitat-dependent differences at the population level in song characteristics, noise levels and territory density. Urban males produced songs with significantly higher peak 
frequencies of the motif and twitter part than conspecifics in the forest $\left(\mathrm{LMM}_{\text {peak }}\right.$ frequency motif: $L \mathrm{ratio}_{1}=22.2, P<0.001$, Fig. 3a and $\mathrm{LMM}_{\text {peak frequency twitter: } L \text { ratio }}=16.4, P<0.001$, Fig. 3d). Temporal song characteristics also differed significantly between urban and forest blackbirds with larger twitter proportions and longer pauses being sung in the city $\left(\mathrm{LMM}_{\text {twitter proportion: }}\right.$ Lratio $_{1}=11.1, P<0.001$, Fig. $3 \mathrm{~g}$ and $\mathrm{LMM}_{\text {pause duration: }}$ Lratio $_{1}=6.3, P=0.01$, Fig. 3j). Furthermore, the habitat in which urban and forest males were singing differed in two ways. Firstly, the territory density of blackbirds was significantly higher in the city than in the forest (Wilcoxon rank-sum test: $W=161, N_{\text {city }}=27, N_{\text {forest }}=$ 24, $P=0.002$, Fig. 3c,f,i,1). Secondly, noise levels in the $1-\mathrm{kHz}$ octave band were significantly higher in the city than in the forest during dawn chorus (Wilcoxon rank-sum test: $\left.W=63, N_{\text {city }}=27, N_{\text {forest }}=24, P<0.001\right)$ even though the noise levels entered into this comparison were taken at a time of the day when there is still relatively little traffic.
Fig. 3 Overview of the song differences between forest males $(n=24)$ and urban males $(n=27)$ and the relationships of song characteristics with noise levels and territory density. The top row shows results for the peak frequency of the motif $(\mathbf{a}-\mathbf{c})$, the second row for the peak frequency of the twitter $(\mathbf{d}-\mathbf{f})$, the third row for the twitter proportion $(\mathbf{g}-\mathbf{i})$ and the bottom row for the pause duration $(\mathbf{j}-\mathbf{l})$. The first column consists of boxplots showing the median (line), interquartile range (box) and $95 \%$ range (whiskers) of the forest population (white bars) and urban population (grey bar) for each of the four song characteristics $(\mathbf{a}, \mathbf{d}, \mathbf{g}, \mathbf{j})$. The middle column has scatterplots showing the noise levels measured during rush hour plotted against the song characteristics of urban males $(\mathbf{b}, \mathbf{e}, \mathbf{h}, \mathbf{k})$. The right column consists of scatterplots showing the territory density plotted against the song characteristics for forest males (white dots, dotted lines) and urban males (grey dots, solid lines) $(\mathbf{c}, \mathbf{f}, \mathbf{i}, \mathbf{l})$. Lines are only shown for significant relationships in the combined analysis of urban and forest males
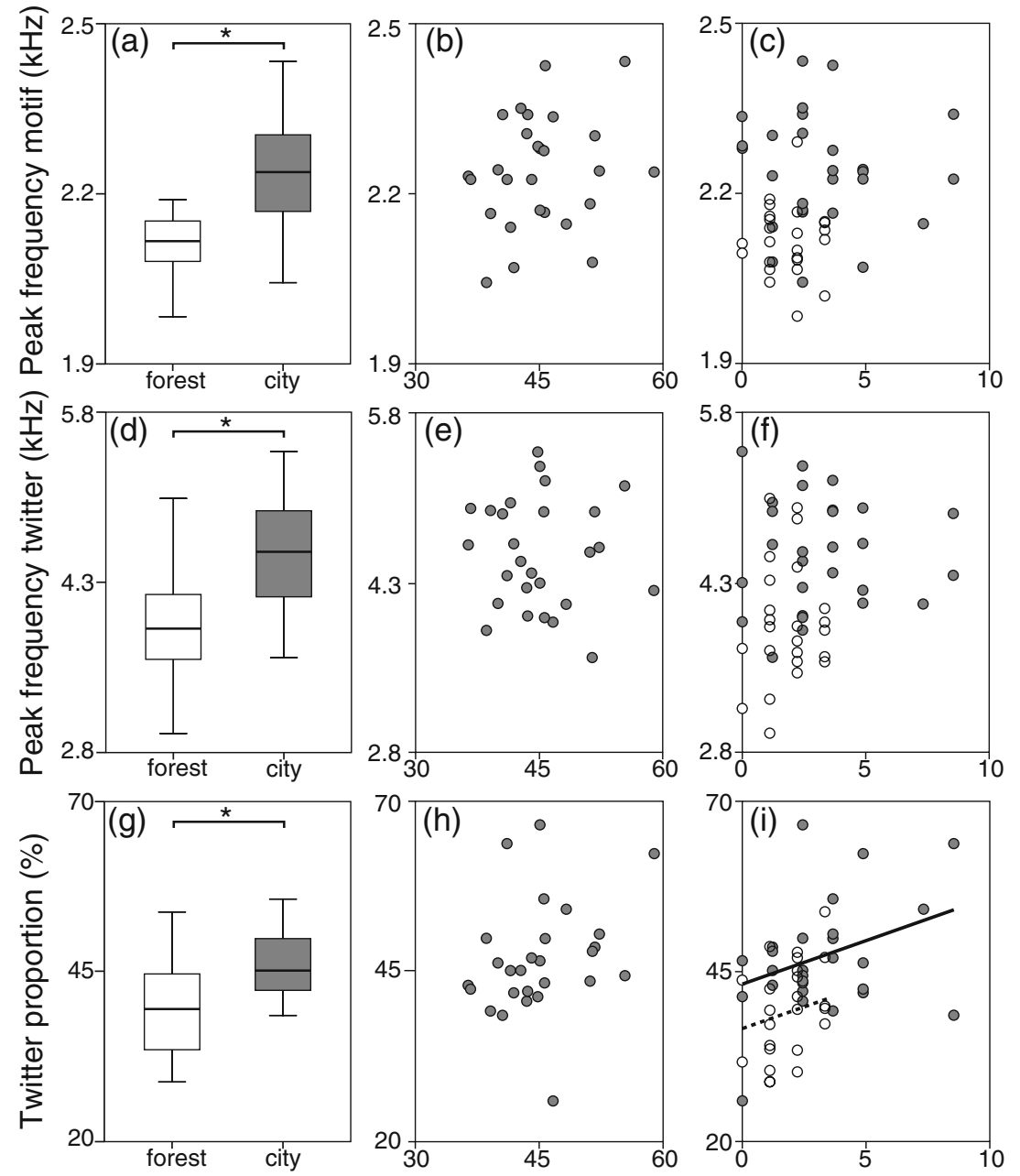

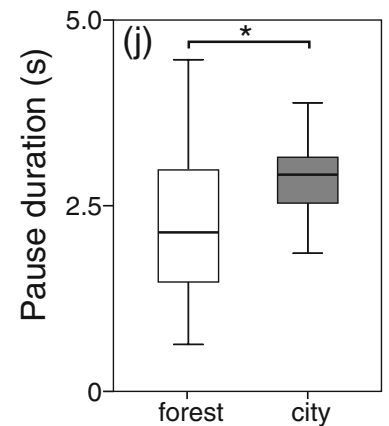

Habitat

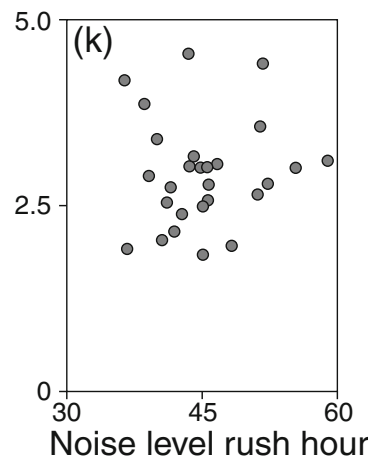

(1 kHz dB) 
Song, territory density and seasonality

In the analysis including data of both the city and the forest, we found several significant relationships between temporal song characteristics and territory density and seasonality. Males in territories with a high number of neighbours produced songs with relatively large twitter proportions and short pauses $\left(\mathrm{LMM}_{\mathrm{twitter}}\right.$ proportion: $L \mathrm{ratio}_{1}=5.4, P=0.02$,

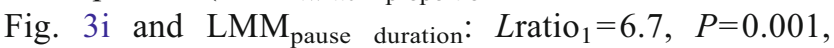
Fig. 31). Furthermore, during the course of the breeding season, the twitter proportion significantly increased $\left(\mathrm{LMM}_{\text {twitter proportion: }} \mathrm{Lratio}_{1}=6.2, P=0.01\right)$ whereas the pause duration stayed the same $\left(\mathrm{LMM}_{\text {pause duration }}: \mathrm{Lratio}_{1}=\right.$ $0.0, P=0.89$ ). Additionally, even with the inclusion of the factors territory density and Julian date in the model, urban males still produced songs with slightly larger twitter proportions and longer pauses than conspecifics in the forest $\left(\mathrm{LMM}_{\text {twitter proportion: }}\right.$ ratio $_{1}=9.7, P=0.002$, Fig. $3 \mathrm{i}$ and $\mathrm{LMM}_{\text {pause duration: }} \operatorname{Lratio}_{1}=11.0, P<0.001$, Fig. 31).

There was no significant effect of territory density on the

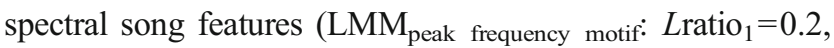

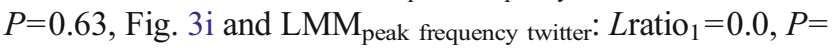
0.85 , Fig. $3 \mathrm{j}$ ). Similarly, Julian date was also not significantly related to the spectral song features $\left(\mathrm{LMM}_{\text {peak frequency motif: }}\right.$ $L_{\text {ratio }}=0.1, P=0.74$ and $\mathrm{LMM}_{\text {peak frequency twitter: } L \text { ratio }}=$ $0.0, P=0.89$ ). As a consequence of the non-significance of territory density and Julian date on spectral song features in these models, habitat had exactly the same effect as in the analyses comparing songs of urban and forest blackbirds at

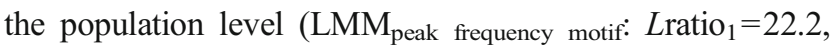

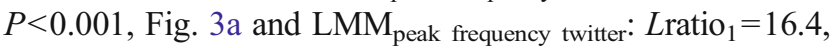
$P<0.001$, Fig. 3d).

Song, noise and seasonality

In the separate analyses for the data of either the city or the forest, we included the noise levels, territory densities and Julian dates in the models. In contrast to the population-level analyses, we found no significant relationship between variation in any of the song characteristics and rush hour noise levels within the urban population $\left(\mathrm{LMM}_{\text {peak frequency motif: }}\right.$ $L$ ratio $_{1}=1.1, P=0.3$, Fig. 3b, $\mathrm{LMM}_{\text {peak }}$ frequency twitter: $L$ ratio $_{1}=0.4, P=0.54$, Fig. 3e, $L_{M M}$ twitter proportion: $L$ ratio $_{1}=$ $0.3, P=0.6$, Fig. $3 \mathrm{~h}$ and $\mathrm{LMM}_{\text {pause duration }}: \mathrm{Lratio}_{1}=0.7$, $P=0.42$, Fig. $3 \mathrm{k})$ nor in the forest population with dawn chorus noise levels $\left(\mathrm{LMM}_{\text {peak }}\right.$ frequency motif: $L$ ratio ${ }_{1}=0.0$, $P=0.84, \mathrm{LMM}_{\text {peak }}$ frequency twitter: $L$ ratio $_{1}=0.0, P=0.94$, LMM twitter proportion: $L \mathrm{Lratio}_{1}=0.0, P=0.87$ and $\mathrm{LMM}_{\text {pause }}$ duration: $L \mathrm{ratio}_{1}=0.8, P=0.38$ ). The temporal song structure varied in the same direction with territory density and Julian date in the city and the forest. The effects were less pronounced than the ones found in the analyses including data of both habitats. In the city, the twitter proportion significantly increased with Julian date (LMM: $L_{\text {ratio }}=5.0$, $P=0.03$ ), and it increased, although not significantly, with territory density (LMM: $L \mathrm{ratio}_{1}=2.8, P=0.09$ ). Pause duration increased in the city with territory density (LMM: $L$ ratio ${ }_{1}=5.0, P=0.03$ ), and no effect of Julian date was observed (LMM: $L$ ratio $_{1}=0.3, P=0.56$ ). In the forest, the twitter proportion significantly increased with Julian date (LMM: $L \mathrm{ratio}_{1}=4.0, P=0.04$ ) and not with territory density (LMM: $L$ ratio $_{1}=1.0, P=0.3$ ). Pause duration in the forest increased, although not significantly, with territory density (LMM: $L$ ratio $_{1}=3.1, P=0.08$ ), and no effect of Julian date was observed (LMM: $L \mathrm{ratio}_{1}=0.2, P=0.67$ ).

\section{Discussion}

Our case study yielded results at two levels. At the population level, we found that the urban and forest population of the European blackbird differed in song, anthropogenic noise levels and territory density. City birds produced songs with a higher peak frequency of the motif and twitter part, larger twitter proportions as well as longer intra-song pauses than conspecifics in the forest (c.f. Ripmeester et al. unpublished). Low-frequency noise levels were louder during dawn chorus in the urban than in the forest territories, which is consistent with the expectations despite the early hour of the dawn chorus relative to the majority of human activities. Singing blackbirds in the city were also surrounded by a larger number of singing neighbours than in the forest, in line with previous reports of relatively high breeding densities in urban environments (Gregoire 2003).

In the analysis comparing variation among individuals, song characteristics were related to territory density and season with respect to temporal, but not spectral features, whereas none of the song characteristics were correlated with noise levels. An increase in territory density coincided with an increase in twitter proportion and shortening of intra-song pause duration. This variation among individuals related to territory density is most likely determined by variation among individuals related to agonistic context (see below). Furthermore, during the course of the breeding season, the twitter proportion became larger. The association of acoustic variation in these temporal features at both the population and individual level indicate that short-term flexibility within individuals related to habitat-dependent territory density and seasonal changes can influence song divergence at the population level.

Song divergence mediated by short-term flexibility

A previous study on three city-forest pairs, including the pair of the current study, showed habitat-dependent song divergence in temporal and spectral features (c.f. Ripmeester 
et al. unpublished). In the present study, we wanted to investigate which factors may be the cause of this divergence and found that temporal song divergence in the investigated city-forest pair is influenced by several factors. Here, we discuss these factors in more detail. Firstly, territory density is higher in the urban than the forest population, and an increase in the number of neighbours is correlated with larger twitter proportions and shorter intra-song pauses. This is in line with the hypothesis that more social interactions lead to larger twitter proportions and shorter pauses within individuals, due to an increased level of arousal (Dabelsteen 1984; Dabelsteen and Pedersen 1990). Secondly, urban blackbirds start to breed several weeks earlier than their conspecifics in the forest (Partecke et al. 2004), and we found in this study that seasonality influences the twitter proportion, as it became larger during the breeding season. These findings corroborate other longitudinal data in which individual males significantly increased their twitter proportions as well as pause durations in a period of several weeks (c.f. Ripmeester et al. unpublished). Seasonal changes in temporal song structure, therefore, seem to reflect intraindividual song variation presumably related to changes in motivational status during the breeding season (Dabelsteen 1984; Dabelsteen and Pedersen 1990).

Our study, thus, suggests that habitat differences in temporal song features can be mediated by short-term flexibility in response to density differences, although we cannot exclude the possibility that part of this divergence is determined by more long-term adaptation. A similar pattern is also found in a study on willow warblers showing that a low- and high-density population differed in temporal song structure, which was suggested to reflect motivational status (Goretskaia 2004). The phenomenon might be widespread as species are likely to occur at different densities in different habitats due to variation in availability of nesting sites and food sources and song variation related to motivation seems a common feature (Nelson 1984; Capp and Searcy 1991; Martin-Vivaldi et al. 2004).

There are also differences between habitats that might lead to song divergence between populations via individual variation due to other factors than motivational state. The Lombard effect, singing louder under more noisy conditions, is a well-known phenomenon in birds (Brumm and Slabbekoorn 2005). Amplitude is a song feature that can vary greatly within individuals, and it has been shown that birds in noisy territories sing louder than conspecifics in quiet territories (Pytte et al. 2003; Brumm 2004). Habitatdependent variation in noise levels could, therefore, translate into differences in song amplitude between populations via intra-individual variation although this has so far not been tested. Nonetheless, experimental studies show that short-term responses to fluctuating noise conditions are not restricted to amplitude adjustments; they are also possible in the temporal domain (Lengagne et al. 1999) and in spectral features (Halfwerk and Slabbekoorn 2009). Intra-individual variation could, therefore, potentially lead to habitat-dependent song divergence in all kinds of song features, related to either motivational status or acoustic conditions, in a wide range of bird species.

Song divergence mediated by non-flexible adaptations

We found no relationship between noise levels and song characteristics at the individual level that could explain song divergence at the population level. Nevertheless, we consider noise still a likely cause for song differentiation as the observed upward shift in song frequency in urban populations relative to forest populations does reduce the average masking effect of anthropogenic noise. Furthermore, our results at the population level match the findings in great tits, the only other species in which songs have been compared in multiple city-forest pairs (Slabbekoorn and den Boer-Visser 2006). Urban great tits also sing consistently higher in cities than in nearby forests, and in contrast to blackbirds, great tits also show a positive relationship at the individual level between anthropogenic noise levels within a territory and the minimum song frequency (Slabbekoorn and Peet 2003). The discrepancy between blackbirds and great tits may be explained by species differences in song-copying behaviour or distinct variation in singing style.

Song-copying behaviour in great tits is closely related to the important role of song matching in territorial interactions (Krebs et al. 1981). Great tits typically produce songs that are copied from territorial neighbours directly around them, which is possible via song learning or repertoire adjustment after dispersal (e.g. Franco and Slabbekoorn 2008). Songs that are better suited to local conditions might, therefore, be selected at the level of the local territory. Blackbirds, on the other hand, do not explicitly use song matching with neighbours and do not exclusively copy songs from direct neighbours. They likely copy from several males in the area during the process of searching and ultimately establishing a territory. When birds copy songs from a relatively large area around their own territory rather than from a restricted set of direct neighbours (e.g. Lachlan and Slater 2003), it is less likely that song features exactly fit environmental conditions in a bird's own territory. This will be particularly the case when the environment is relatively heterogeneous like with varying anthropogenic noise levels in cities.

Different singing styles may be another explanation for the discrepancy in noise-related acoustic variation between European blackbirds and great tits. Male great tits sing with eventual variety, repeating the same song type several times 
before switching to another, which may facilitate cultural selection of locally adapted song. During repetition of a single song type, great tits might get auditory or social feedback on the suitability of that particular song type. The existence and impact of such a mechanism is supported by a recent experimental study showing noise-dependent song frequency use by great tits that continue to produce a song type for longer when it does relatively well under current noise conditions (Halfwerk and Slabbekoorn 2009). In contrast to great tits, blackbirds have a large repertoire from which they produce songs with immediate variety, changing song type every consecutive song. This might make it more difficult to adjust repertoire use to local conditions via an immediate feedback mechanism. This hypothesis is supported by the observation that blackbird song frequencies do not or hardly change within individuals with naturally fluctuating noise conditions (c.f. Ripmeester et al. unpublished).

In conclusion, we have shown that habitat-related song divergence at the population level can be mediated by factors that are only indirectly related to habitat characteristics and that may emerge through short-term flexibility in singing behaviour. Temporal variation in blackbird song at the individual level, related to territory density and season, explained at least part of the temporal variation at the population level. Such an impact may undermine the potential role for song as a population marker in communication with competing males or potential mates. Consequently, studies on the impact of song on hybridisation avoidance and speciation require insight into the contribution of short-term flexibility to habitat-related patterns of acoustic divergence. This applies to birds but may very well be critical for a wider taxonomic range including for example frogs and primates (Ryan et al. 1990; Sugiura et al. 2006; Koda et al. 2008; Schwartz et al. 2008). Furthermore, we found a mismatch between populationand individual-level correlations in noise-dependent frequency use for blackbirds which is in contrast to results obtained in great tits. This suggests the existence of species-specific causal explanations for patterns of acoustic variation among individuals and among populations, which are possibly related to song-copying behaviour or feedback constraints related to singing style. Therefore, more taxonomic replicates are needed to confirm whether the differences between blackbirds and great tits reflect some kind of general categories in variable patterns of noisedependent acoustic variation in birdsong.

Acknowledgments We thank Carel ten Cate for helpful comments on the manuscript, Peter Snelderwaard for technical support, Tom van Dooren and Caroline van Heijningen for statistical advice and Duinwaterbedrijf Zuid-Holland for permission to conduct fieldwork in Meijendel.
Open Access This article is distributed under the terms of the Creative Commons Attribution Noncommercial License which permits any noncommercial use, distribution, and reproduction in any medium, provided the original author(s) and source are credited.

\section{References}

Brumm H (2004) The impact of environmental noise on song amplitude in a territorial bird. J Anim Ecol 73:434-440

Brumm H, Slabbekoorn H (2005) Acoustic communication in noise. Adv Study Behav 35:151-209

Capp MS, Searcy WA (1991) An experimental-study of song type function in the bobolink (Dolichonyx oryzivorus). Behav Ecol Sociobiol 28:179-186

Clement P, Hathway R (2000) Helm identification guides: thrushes. Christopher Helm Ltd, London

Dabelsteen T (1984) An analysis of the full song of the blackbird Turdus merula with respect to message coding and adaptations for acoustic communication. Ornis Scand 15:227-239

Dabelsteen T, Pedersen SB (1985) Correspondence between messages in the full song of the blackbird Turdus merula and meanings to territorial males, as inferred from responses to computerized modifications of natural song. Z Tierpsychol 69:149-165

Dabelsteen T, Pedersen SB (1990) Song and information about aggressive responses of blackbirds, Turdus merula: evidence from interactive playback experiments with territory owners. Anim Behav 40:1158-1168

de Vos D, de Meersman L (2005) Wat zingt daar? KNNV Uitgeverij, Utrecht

Dingle C, Halfwerk W, Slabbekoorn H (2008) Habitat-dependent song divergence at subspecies level in the grey-breasted wood-wren. J Evol Biol 21:1079-1089

Ellers J, Slabbekoorn H (2003) Song divergence and male dispersal among bird populations: a spatially explicit model testing the role of vocal learning. Anim Behav 65:671-681

Fernández-Juricic E, Poston R, De Collibus K, Morgan T, Bastain B, Martin C, Jones K, Treminio R (2005) Microhabitat selection and singing behavior patterns of male house finches (Carpodacus mexicanus) in urban parks in a heavily urbanized landscape in the Western U.S. Urban Habitats 3:49-69

Fernández-Juricic E, del Nevo AJ, Poston R (2009) Identification of individual and population-level variation in vocalizations of the endangered southwestern willow flycatcher (Empidonax traillii extimus). Auk 126:89-99

Franco P, Slabbekoorn H (2008) Repertoire size and composition in great tits: a flexibility test using playbacks. Anim Behav 77:261269

Goretskaia MI (2004) Song structure and singing behaviour of willow warbler Prylloscopus trochilus acredula in populations of low and high density. Bioacoustics-the International Journal of Animal Sound and Its Recording 14:183-195

Gregoire A (2003) Démographie et différenciation chez le Merle noir Turdus merula: liens avec l'habitat et les relations hôtes-parasites. In. University of Bourgogne, Dijon, p 212

Halfwerk W, Slabbekoorn H (2009) A behavioural mechanism explaining noise-dependent frequency use in urban bird song. Anim Behav, in press

Hustings F, Vergeer JW (2002) Atlas van de Nederlandse broedvogels. verspreiding, aantallen, verandering. KNNV Uitgeverij, Utrecht

Ihaka R, Gentleman R (1996) R: A language for data analysis and graphics. J Comput Graph Stat 5:299-314 
Koda H, Shimooka Y, Sugiura H (2008) Effects of caller activity and habitat visibility on contact call rate of wild Japanese Macaques (Macaca fuscata). Am J Primatol 70:1055-1063

Krebs JR, Ashcroft R, Vanorsdol K (1981) Song matching in the great tit Parus major. Anim Behav 29:918-923

Kroodsma DE (2004) Diversity and plasticity of bird song. In: Marler P, Slabbekoorn H (eds) Nature's music: the science of birdsong. Elsevier, London, pp 108-130

Lachlan RF, Slater PJB (2003) Song learning by chaffinches: how accurate, and from where? Anim Behav 65:957-969

Lengagne T, Aubin T, Lauga J, Jouventin P (1999) How do king penguins (Aptenodytes patagonicus) apply the mathematical theory of information to communicate in windy conditions? Proc R Soc Lond B Biol Sci 266:1623-1628

Luniak M, Mulsow R, Walasz K (1990) Urbanization of the European blackbird: expansion and adaptations of urban populations. In: Urban ecological studies in Central and Eastern Europe; international symposium Warsaw. Polish Academy of Sciences, Warsaw, pp 187-198

Martin-Vivaldi M, Palomino JJ, Soler M (2004) Strophe length in spontaneous songs predicts male response to playback in the hoopoe Upupa epops. Ethology 110:351-362

Morton ES (1975) Ecological sources of selection on avian sounds. Am Nat 109:17-34

Mundinger PC (1982) Microgeographic and macrogeographic variation in the acquired vocalizations of birds. In: Kroodsma DE, Miller EH (eds) Acoustic communication in birds: song learning and its consequences, vol 2. Academic, New York, pp 147-208

Nelson DA (1984) Communication of intentions in agonistic contexts by the pigeon guillemot, Cepphus columba. Behaviour 88:145-189

Partecke J, Van't Hof T, Gwinner E (2004) Differences in the timing of reproduction between urban and forest European blackbirds
(Turdus merula): result of phenotypic flexibility or genetic differences? Proc R Soc Lond B Biol Sci 271:1995-2001

Podos J, Nowicki S (2004) Beaks, adaptation, and vocal evolution in Darwin's finches. Bioscience 54:501-510

Pytte CL, Rusch KM, Ficken MS (2003) Regulation of vocal amplitude by the blue-throated hummingbird, Lampornis clemenciae. Anim Behav 66:703-710

Ripmeester EAP, de Vries AM, Slabbekoorn H (2007) Do blackbirds signal motivation to fight with their song? Ethology 113:1021-1028

Ryan MJ, Cocroft RB, Wilczynski W (1990) The role of environmental selection in intraspecific divergence of mate recognition signals in the cricket frog, Acris crepitans. Evolution 44:1869-1872

Schwartz JJ, Brown R, Turner S, Dushaj K, Castano M (2008) Interference risk and the function of dynamic shifts in calling in the gray treefrog (Hyla versicolor). J Comp Psychol 122:283-288

Slabbekoorn H, den Boer-Visser A (2006) Cities change the songs of birds. Curr Biol 16:2326-2331

Slabbekoorn H, Peet M (2003) Birds sing at a higher pitch in urban noise. Nature 424:267

Slabbekoorn H, Smith TB (2002a) Bird song, ecology and speciation. Philos Trans R Soc B-Biol Sci 357:493-503

Slabbekoorn H, Smith TB (2002b) Habitat-dependent song divergence in the little greenbul: an analysis of environmental selection pressures on acoustic signals. Evolution 56:1849-1858

Slabbekoorn H, Yeh P, Hunt K (2007) Sound transmission and song divergence: a comparison of urban and forest acoustics. Condor 109:67-78

Sugiura H, Tanaka T, Masataka N (2006) Sound transmission in the habitats of Japanese macaques and its possible effect on population differences in coo calls. Behaviour 143:993-1012

Wood WE, Yezerinac SM (2006) Song sparrow (Melospiza melodia) song varies with urban noise. Auk 123:650-659 